\title{
The Long-Term Burden of COPD Exacerbations During Maintenance Therapy and Lung Function Decline
}

This article was published in the following Dove Press journal: International Journal of Chronic Obstructive Pulmonary Disease

\author{
Marjan Kerkhof $\mathbb{D}^{1}$ \\ Jaco Voorham (ID) \\ Paul Dorinsky (iD ${ }^{2}$ \\ Claudia Cabrera ${ }^{3,4}$ \\ Patrick Darken ${ }^{5}$ \\ Janwillem WH Kocks ${ }^{1,6}$ \\ Mohsen Sadatsafavi $\mathbb{D D}^{7,8}$ \\ Don D Sin $\mathbb{D}^{7,8}$ \\ Victoria Carter ${ }^{\prime}$ \\ David B Price (D) ${ }^{1,9}$ \\ 'Observational \& Pragmatic Research \\ Institute Pte Ltd, Singapore, Singapore; \\ ${ }^{2}$ AstraZeneca, Durham, NC, USA; \\ ${ }^{3}$ AstraZeneca, Mölndal, Sweden; \\ ${ }^{4}$ Department of Medical Epidemiology \\ and Biostatistics, Karolinska Institute, \\ Stockholm, Sweden; ${ }^{5}$ AstraZeneca, \\ Morristown, NJ, USA; ${ }^{6}$ General \\ Practitioners Research Institute, \\ Groningen, The Netherlands; \\ ${ }^{7}$ Respiratory Evaluation Sciences \\ Program, Collaboration for Outcomes \\ Research and Evaluation, University of \\ British Columbia, Vancouver, BC, \\ Canada; ${ }^{8}$ Centre for Heart Lung \\ Innovation, St. Paul's Hospital, Vancouver, \\ BC, Canada; ${ }^{9}$ Academic Primary Care, \\ University of Aberdeen, Aberdeen, UK
}

Correspondence: David B Price Polwarth Building, Foresterhill, Aberdeen AB25 2ZD, UK

Tel +656962 3627

Email dprice@opri.sg
Introduction: Early identification of preventable risk factors of COPD progression is important. Whether exacerbations have a negative impact on disease progression is largely unknown. We investigated whether the long-term occurrence of exacerbations is associated with lung function decline at early stages of COPD.

Methods: Patients diagnosed with mild/moderate COPD (obstruction and $\mathrm{FEV}_{1} \%$ predicted $50-90 \%$ ), aged $\geq 35$ years, and a smoking history, who had $\geq 6$ years of UK electronic medical records after initiation of maintenance therapy were studied. Multilevel mixedeffect linear regression was performed to determine the association between the count of any year in which the patient had $\geq 1$ exacerbation over a 6-year period and $\mathrm{FEV}_{1}$ decline, adjusted for sex, age, anthropometrics and smoking habits. Exacerbations were defined as any prescription for an acute oral corticosteroid course and/or lower respiratory-related antibiotics and/or any COPD-related emergency or inpatient hospitalization.

Results: Of 11,337 patients included (mean age 65 years; 49\% female) $31.6 \%, 23.3 \%$, $16.6 \%, 11.6 \%, 8.1 \%, 5.3 \%$ and $3.4 \%$ had $0,1,2,3,4,5$ and 6 years with $\geq 1$ exacerbation. The mean annual $\mathrm{FEV}_{1}$ decline accelerated by $1.50 \mathrm{~mL} /$ year $(95 \%$ Confidence Interval 1.02; 1.98 ) with every additional year with $\geq 1$ exacerbation from $31.0 \mathrm{~mL} /$ year in subjects without any exacerbation to $40.0 \mathrm{~mL} /$ year in patients experiencing $\geq 1$ exacerbation every year. Patients with more years with $\geq 1$ exacerbation had a lower mean $\mathrm{FEV}_{1}$ at first diagnosis: $14.7 \mathrm{~mL}(11.7 ; 17.8)$ lower with every additional year with exacerbations. When counting years with $\geq 2$ exacerbations, greater effects were observed $(2.19[1.50 ; 2.88] \mathrm{mL} /$ year excess decline per year with $\geq 2$ exacerbations; $16.5 \mathrm{~mL}$ [12.1; 20.8] lower $\mathrm{FEV}_{1}$ at diagnosis).

Conclusion: Patients who experienced a greater exacerbation burden after initiation of maintenance therapy had worse lung function at diagnosis and a more rapid lung function decline thereafter, which emphasizes the need for better treatment strategies.

Keywords: COPD, exacerbations, spirometry, inhalation therapy, observational study

\section{Plain Language Summary}

Patients with chronic obstructive pulmonary disease (COPD) may experience exacerbations, episodes of acute worsening of symptoms (also known as lung attacks), which may deteriorate lung function. Maintenance inhalation therapy may prevent exacerbations in some patients, but many patients continue experiencing exacerbations despite therapy. This study was conducted to investigate whether information on the long-term exacerbation burden, measured by counting the number of years over a 6-year period that patients had exacerbations despite maintenance therapy, can help identifying patients with more accelerated lung function decline at early stages of COPD. Using data from two large electronic medical record databases in the UK, the researchers found that 
patients with more exacerbations after initiation of maintenance therapy had worse lung function at the time of first diagnosis and a more rapid lung function decline thereafter. The results, therefore, emphasize the need for more precise and aggressive intervention at an earlier stage of COPD to delay lung function loss.

\section{Introduction}

Chronic obstructive pulmonary disease (COPD) is a chronic and debilitating respiratory disease characterized by progressively declining irreversible airflow limitation. It constitutes a global public health burden, associated with substantial morbidity and mortality. ${ }^{1}$ The WHO estimated that 65 million people have moderate-severe COPD, accounting for $5 \%$ of all causes of death in 2005 . $^{2}$

COPD is a heterogeneous condition with a high degree of variation in the clinical presentation and rate of disease progression among individuals. Early life exposures and smoking are important factors predicting lung function in adult life. ${ }^{1}$ However, why in some patients lung function declines rapidly and in others not is not well understood. ${ }^{3}$ In particular, the role of the burden of COPD in terms of occurrence of exacerbations, events of acute worsening of COPD symptoms, on disease progression needs to be further studied. Whether a high exacerbation burden early in the disease may lead to accelerated decline has not been fully elucidated. However, there is sparse evidence that COPD exacerbations are associated with subsequent accelerated lung function decline, particularly in those with mild disease. ${ }^{4}$ Lung function decline is most accelerated at the early stages of $\mathrm{COPD}^{5,6}$ and early identification of patients at risk followed by effective treatment of exacerbations may therefore be important.

Understanding the impact of treatment on exacerbation burden and ultimately disease progression was thus the aim of this study. We studied whether the long-term burden of COPD exacerbations occurring after initiation of maintenance therapy is associated with lung function decline at early stages of COPD.

\section{Methods}

\section{Data Source}

This is a historical cohort study using primary care data from the Optimum Patient Care Research Database (OPCRD; https://opcrd.co.uk/) and the Clinical Practice Research Datalink (CPRD (protocol number 17_117); http://www.cprd.com/). The OPCRD is an anonymized, longitudinal medical record database of over 8 million patients from 700 primary care practices across the UK with a linked patient-completed respiratory questionnaire. ${ }^{7}$
It houses high-quality, validated data used regularly in clinical, epidemiological, and pharmaceutical research and is approved by the Health Research Authority of the UK National Health Service for clinical research use (Research Ethics Committee reference: 15/EM/0150). The CPRD is a well-validated computerized database frequently used for medical and health research, containing de-identified, longitudinal medical records of over 11 million active patients from UK primary care practices. Coded diagnosis of COPD within the databases utilized that of Quality and Outcomes Framework (QOF) which has been previously validated. ${ }^{8}$

\section{Study Design and Patients}

Patient inclusion and exclusion criteria for this study are detailed in Table 1. The list of Read Codes used in this study is available upon request.

Table I Inclusion and Exclusion Criteria

\section{Inclusion Criteria}

- Diagnostic Read Code (Coding system used in the UK) for COPD.

- Valid spirometry recorded in the period between 3 months before and 12 months after the first diagnostic Read code for COPD. Date of the spirometry designated as the index date.

- Evidence of obstruction ever recorded, ie, $\mathrm{FEV}_{\mathrm{l}} /$ /Forced Vital Capacity $(\mathrm{FVC})<0.7$.

- Mild to moderate airflow limitation at the time of first clinical diagnosis, ie, $\mathrm{FEV}_{1} \%$ predicted $\geq 50 \%$ and $\leq 90 \%$ at index date, as there is limited scope for further FEV, decline and early intervention in patients with more severe airflow limitation.

- Age $\geq 35$ years

- $\geq 1$ year of continuous medical records prior to the index date for baseline characterization

- $\geq 6$ years of continuous medical records following the start of any maintenance therapy

- $\geq I$ FEV, recording after index date

- A history of tobacco smoking (ex or current) at the time of first COPD diagnosis, ie, at any recording of smoking status prior to diagnosis or at first recording after diagnosis

- Height recorded within 5 years of index date and weight at adult age ( $\geq 21$ years)

Exclusion criteria

- Active asthma at or after the date of first COPD diagnosis, defined as $\geq$ I diagnostic Read code for asthma, or $\geq I$ asthma monitoring or review code recorded, or asthma-COPD overlap syndrome as first COPD diagnostic code or recorded during follow-up

- Diagnostic code for other chronic lower respiratory conditions recorded ever 
All recorded $\mathrm{FEV}_{1}$ values were used to estimate lung function decline during follow-up. The date of the $\mathrm{FEV}_{1}$ recording at time of diagnosis (defined as date of spirometry within 3 months before to 12 months after COPD diagnosis) was chosen as the index date to characterize patients. At least one year of data prior to the index date was used for baseline characterization.

The primary outcome was the annual decline in $\mathrm{FEV}_{1}$ during spirometry follow-up, expressed in $\mathrm{mL}$ per year. $\mathrm{FEV}_{1}$ (in $\mathrm{mL}$ ) at index date (around time of first recorded clinical COPD diagnosis) was a secondary outcome.

We studied the association between the count of any year in which the patient had $\geq 1$ exacerbation over a 6-year period following the date at which maintenance therapy was initiated and the outcomes. Maintenance therapy included any of the following therapy prescribed during follow-up as a single inhaler or in combination: inhaled corticosteroids (ICS), long-acting $\beta 2$-agonist (LABA) or long-acting muscarinic antagonist (LAMA).

Exacerbations were defined as a prescription for acute oral corticosteroids and/or a respiratory-related antibiotic course and/or an emergency or inpatient hospitalization. Analyses were also performed for the number of years with frequent exacerbations $(\geq 2$ exacerbations per year, as per GOLD definition). ${ }^{1}$

\section{Statistical Analysis}

Statistical analyses were performed in Stata version 14.2 (StataCorp, College Station, TX). Descriptive statistics were summarized for baseline characteristics. Categorical variables were presented as numbers and proportions and continuous variables as mean with standard deviation or median with interquartile range.

A multilevel 'random-slope/random-intercept' model was used to model $\mathrm{FEV}_{1}$ change over time. Fixed effects with $95 \%$ confidence intervals were estimated for the number of years with $\geq 1$ exacerbation, both analyzed as categorical $(0,1,2,3,4,5,6)$ and continuous variable (with the latter showing a slightly better model fit when calculated as Akaike Information Criterion (AIC)). The associations with both intercept $\left(\mathrm{FEV}_{1}\right.$ at index date, ie, spirometry at time of COPD diagnosis) and slope ( $\mathrm{FEV}_{1}$ decline over time) were studied. An interaction term of follow-up time with the number of years with exacerbations represented the association of exposure with slope of $\mathrm{FEV}_{1}$ decline. Random effects were included allowing each patient's change in lung function over time to be scattered around the population average trajectory, assuming that the errors were independent and normally distributed. The model also included sex, age, height, weight, and smoking status as covariates, including an interaction term with time, to adjust for potential confounding by other well-known risk factors of lung function decline. ${ }^{9}$ We have also tested whether the presence of comorbidities changed the associations by including the variables into the model separately $(<2 \%$ change in coefficient). Mean marginal $\mathrm{FEV}_{1}$ decline and $\mathrm{FEV}_{1}$ at index date with $95 \%$ confidence intervals were visualized for each possible number of years the patients experienced any exacerbation within the 6 years of follow-up analyzing a categorical variable.

Given the recent, albeit controversial evidence on the effect of ICS on slope of decline we also performed analyses including an interaction term with a time-varying variable indicating whether the most recent therapy prior to the lung function measurement (within previous 120 days) contained ICS or not.

Sensitivity analyses were conducted excluding $\mathrm{FEV}_{1}$ measurements taken within 2 weeks before or after the occurrence of an exacerbation and excluding patients who ever had a diagnosis of asthma prior to index date..$^{10,11}$

\section{Results}

\section{Patient Characteristics}

The flowchart for patient selection is depicted in Figure 1. Following exclusion and inclusion criteria, 11,337 patients who had at least 6 years of electronic medical data since initiation of maintenance therapy were identified. Of these, $43 \%$ had already started inhaler maintenance therapy at index date and $42 \%$ started in the first year afterwards.

Patient demographics are shown in Table 2 and Supplementary Table E1. Patients were on average 65 years old at index date $\left(\mathrm{FEV}_{1}\right.$ around time of first COPD diagnosis) with almost even distribution of gender $(51 \%$ men). A total of $52.4 \%$ of patients were indicated as exsmokers in the baseline period; $31 \%$ continued smoking, and $36 \%$ were sustained quitters after index date.

Most patients had no (31.5\%) or only one year $(23.2 \%)$ with $\geq 1$ exacerbation in the first 6 years after therapy initiation (Figure 2). A total of $45.1 \%, 28.5 \%, 16.9 \%$, $8.7 \%$ and $3.4 \%$ had $\geq 2, \geq 3, \geq 4, \geq 5$ and $\geq 6$ years with $\geq 1$ exacerbation, respectively (mean ( \pm standard deviation): $1.7( \pm 1.7)$ years $)$.

A total of $35 \%$ of patients experienced at least one year with frequent exacerbations ( $\geq 2$ exacerbations per year) 


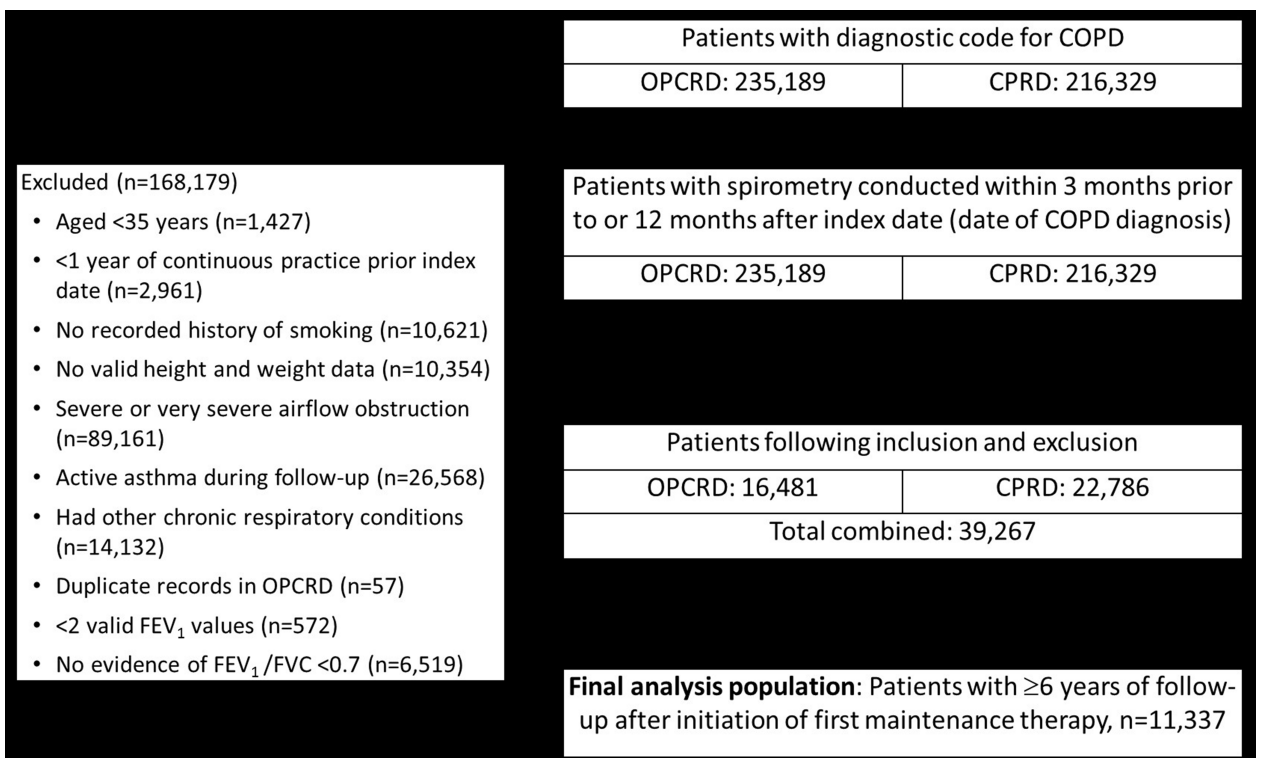

Figure I Patient selection flowchart.

during the first 6 years after maintenance therapy, and $0.8 \%$ experienced frequent exacerbations in every year throughout the 6 years (Figure 2).

\section{Association Between the Number of Years with Exacerbation and FEV, Decline}

Compared to patients without exacerbations, mean $\mathrm{FEV}_{1}$ decline over time was greater for patients who experienced exacerbations after initiation of maintenance treatment (Figure 3). The mean annual $\mathrm{FEV}_{1}$ decline accelerated by $1.50 \mathrm{~mL} /$ year (95\% Confidence Interval $1.02 ; 1.98, \mathrm{p}<0.001$ ) with every additional year with $\geq 1$ exacerbation from $31.0 \mathrm{~mL} /$ year in subjects without any exacerbation to $40.0 \mathrm{~mL} /$ year in patients experiencing $\geq 1$ exacerbation every year, when analyzed as a continuous variable. A greater effect was observed when counting the number of years with multiple $(\geq 2)$ exacerbations with a $2.19 \mathrm{~mL} /$ year $(95 \%$ CI $1.51 ; 2.88, \mathrm{p}<0.001)$ excess decline with every one year increase in experiencing $\geq 2$ exacerbations.

\section{Association Between the Number of Years with Exacerbation Events and Fev, at the Time of First Clinical COPD Diagnosis}

Patients with more years with $\geq 1$ exacerbation had a lower mean $\mathrm{FEV}_{1}$ at first diagnosis: $14.7 \mathrm{~mL}(11.7 ; 17.8, \mathrm{p}<0.001)$ lower with every additional year with $\geq 1$ exacerbation.
When counting years with $\geq 2$ exacerbations, $\mathrm{FEV}_{1}$ at index date was $16.5 \mathrm{~mL}(12.1 ; 20.8, \mathrm{p}<0.001)$ lower for every additional year with $\geq 2$ exacerbations) (Figure 4 ).

\section{Treatment with or without ICS During Follow-Up}

Of 82,956 spirometry measurements recorded, $52 \%$ were performed after a recent prescription for ICS; $35 \%$ of patients already had ICS prescribed prior to spirometry performed around first diagnosis. Recent ICS therapy did not influence the observed acceleration of $\mathrm{FEV}_{1}$ decline with an increasing number of years with exacerbation ( $p$-value for interaction term of time with the continuous variable of number of years with $\geq 1$ exacerbation and presence of ICS prescription within 120 days prior to $\mathrm{FEV}_{1}$ measurement $\left.=0.961\right)($ Supplementary Figure E1 $)$. However, treatment with ICS was associated with a slightly higher $\mathrm{FEV}_{1}$ at the time of first COPD diagnosis, in particular for patients who had no exacerbations after initiating maintenance therapy $(31 \mathrm{~mL}$ ( $95 \%$ confidence interval: 23 to 39 ) higher when treated with ICS) (p-value interaction term of intercept with variables $=0.051$ ) (Supplementary Figure E1).

We have found no change in associations after adjustment for baseline comorbidities diagnosed ever.

\section{Sensitivity Analyses}

A sensitivity analysis excluding 5647 (6.8\%) FEV $_{1}$ values recorded within a window of 2 weeks before or 
Table 2 Patient Characteristics

\begin{tabular}{|c|c|c|}
\hline \multicolumn{2}{|l|}{ Variable } & \multirow{2}{*}{$\begin{array}{l}\text { Total }(\mathbf{N}=11,337) \\
8.2(7.0 ; 10.1)\end{array}$} \\
\hline Spirometry follow-up after index date (ID), years & Median (IQR) & \\
\hline Age (years) at index date & Mean (SD) & $65.2(10.0)$ \\
\hline Sex & Male, n (\%) & $5771(50.9)$ \\
\hline Body mass index, closest to index date & $\begin{array}{l}\text { Mean (SD) } \\
\text { Underweight, n (\%) } \\
\text { Normal Weight, n (\%) } \\
\text { Overweight, n (\%) } \\
\text { Obese, n (\%) }\end{array}$ & $\begin{array}{l}27.4(5.6) \\
329(2.9) \\
3656(32.2) \\
4143(36.5) \\
3209(28.3)\end{array}$ \\
\hline Baseline smoking status, closest to index date & $\begin{array}{l}\mathrm{N}(\% \text { non-missing) } \\
\text { Ex-smoker, } \mathrm{n}(\%)\end{array}$ & $\begin{array}{l}10,411(91.8) \\
5454(52.4)\end{array}$ \\
\hline Smoking status up to 5 years of follow-up after index date & $\begin{array}{l}\mathrm{N}(\% \text { non-missing) } \\
\text { Sustained quitter, } \mathrm{n}(\%) \\
\text { Intermittent quitter, n (\%) } \\
\text { Continuous smoker, n (\%) }\end{array}$ & $\begin{array}{l}10,462(92.3) \\
3785(36.2) \\
3467(33.1) \\
3210(30.7)\end{array}$ \\
\hline Asthma diagnosis prior to COPD diagnosis & Ever, n (\%) & $1423(12.6)$ \\
\hline GOLD stage of airflow limitation at index date & $\begin{array}{l}\text { GOLD 2: (moderate: } 50 \% \leq \mathrm{FEV}_{\text {, }} \\
<80 \%) \text {, n }(\%)\end{array}$ & $10,116(89.2)$ \\
\hline mMRC dyspnea scale & $\begin{array}{l}\mathrm{N}(\% \text { non-missing) } \\
0, \mathrm{n}(\%) \\
\mathrm{I}, \mathrm{n}(\%) \\
2, \mathrm{n}(\%) \\
3, \mathrm{n}(\%) \\
4, \mathrm{n}(\%)\end{array}$ & $\begin{array}{l}10,438(92.1) \\
1913(18.3) \\
5058(48.5) \\
2513(24.1) \\
833(8.0) \\
121(1.2)\end{array}$ \\
\hline $\begin{array}{l}\text { GOLD group ( } 2017 \text { definition, based on } \mathrm{mMRC} \text { and exacerbations in last year) } \\
\text { at index date }\end{array}$ & $\begin{array}{l}\text { N (\% non-missing) } \\
\text { A, n (\%) } \\
\text { B, n (\%) } \\
\text { C, n (\%) } \\
\text { D, n (\%) }\end{array}$ & $\begin{array}{l}10,438(92.1) \\
564 \mid(54.0) \\
2762(26.5) \\
1330(12.7) \\
705(6.8)\end{array}$ \\
\hline COPD exacerbations in year prior to index date & $\begin{array}{l}\text { Mean (SD) } \\
0, n(\%) \\
I, n(\%) \\
2, n(\%) \\
3, n(\%) \\
\geq 4, n(\%)\end{array}$ & $\begin{array}{l}0.8(1.1) \\
6298(55.6) \\
2946(26.0) \\
1241(10.9) \\
505(4.5) \\
347(3.1)\end{array}$ \\
\hline Highest level of maintenance therapy initiated during follow-up & $\begin{array}{l}\text { LAMA or LABA } \\
\text { ICS monotherapy } \\
\text { ICS+(LABA or LAMA) } \\
\text { LABA+LAMA } \\
\text { Triple therapy }\end{array}$ & $\begin{array}{l}593(5.2) \\
1081(9.5) \\
2901(25.6) \\
462(4.1) \\
6300(55.6)\end{array}$ \\
\hline $\begin{array}{l}\text { Blood eosinophil count, } \times 10^{9} \text { cells/liter, closest within } 2 \text { years of highest } \\
\text { maintenance therapy initiation }\end{array}$ & $\begin{array}{l}\mathrm{N}(\% \text { non-missing) } \\
<0.15, \mathrm{n} \mathrm{( \% )} \\
0.15-0.34, \mathrm{n}(\%) \\
\geq 0.35, \mathrm{n} \mathrm{( \% )}\end{array}$ & $\begin{array}{l}6171(54.4) \\
1924(31.2) \\
3062(49.6) \\
1185(19.2)\end{array}$ \\
\hline
\end{tabular}

Abbreviations: IQR, interquartile range; SD, standard deviation; mMRC, modified medical research council; GOLD, global initiatives for chronic obstructive lung disease; $\mathrm{FEV}_{\mathrm{I}}$, forced expiratory volume in one second. 


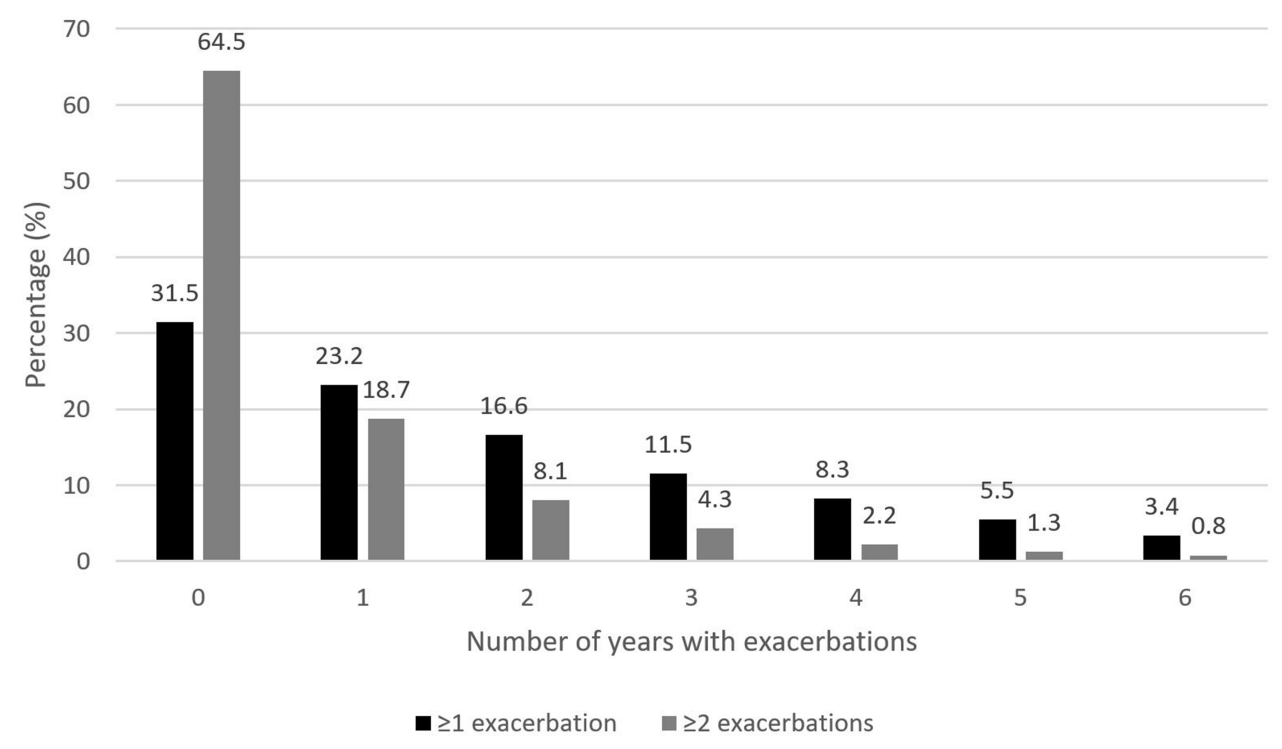

Figure 2 Distribution of the number of years with $\geq 1$ and $\geq 2$ COPD exacerbations after initiation of maintenance therapy.
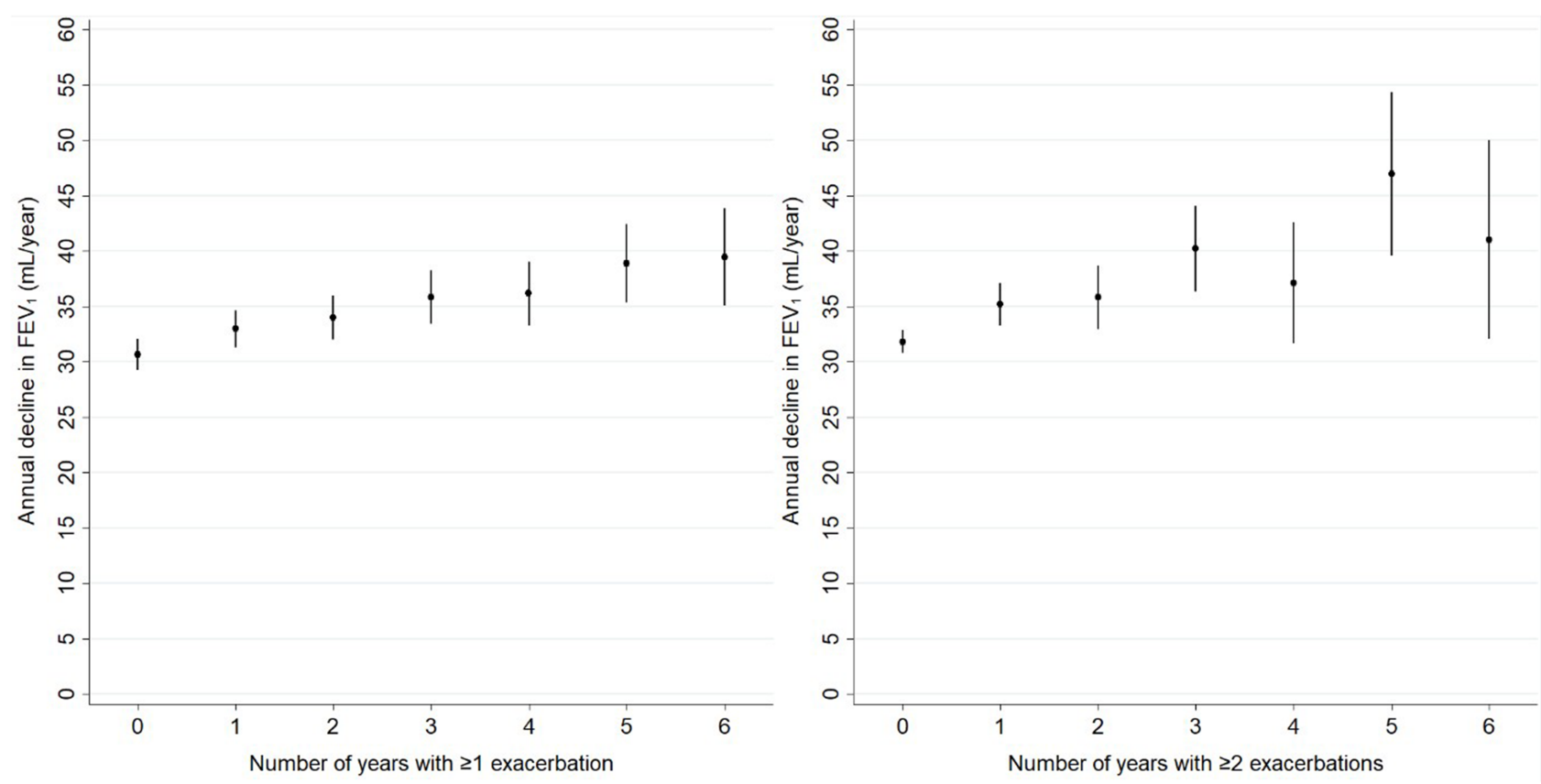

Figure 3 Marginal mean decline in $\mathrm{FEV}_{\mathrm{I}}$ (mL/year) with 95\% confidence interval by the number of years patients experienced $\geq \mathrm{I}$ (left) and $\geq 2$ (right) COPD exacerbations during 6 years after initiation of maintenance therapy.

after the occurrence of an exacerbation showed similar results for the association of years with $\geq 1$ exacerbation with the rate of $\mathrm{FEV}_{1}$ decline and $\mathrm{FEV}_{1}$ at index date (Supplementary Figure E2). No relevant differences in results were observed after excluding 1423 (12.6\%) patients who ever had a diagnosis of asthma recorded prior to the baseline spirometry. (Supplementary Figure E3).

\section{Discussion}

\section{Summary of Findings}

This study is, to the extent of our knowledge, the first to show that a higher long-term burden of COPD exacerbations while receiving maintenance treatment is associated with accelerated lung function decline over time. Patients with a higher exacerbation burden already had a lower lung function at the time of first clinical diagnosis of 

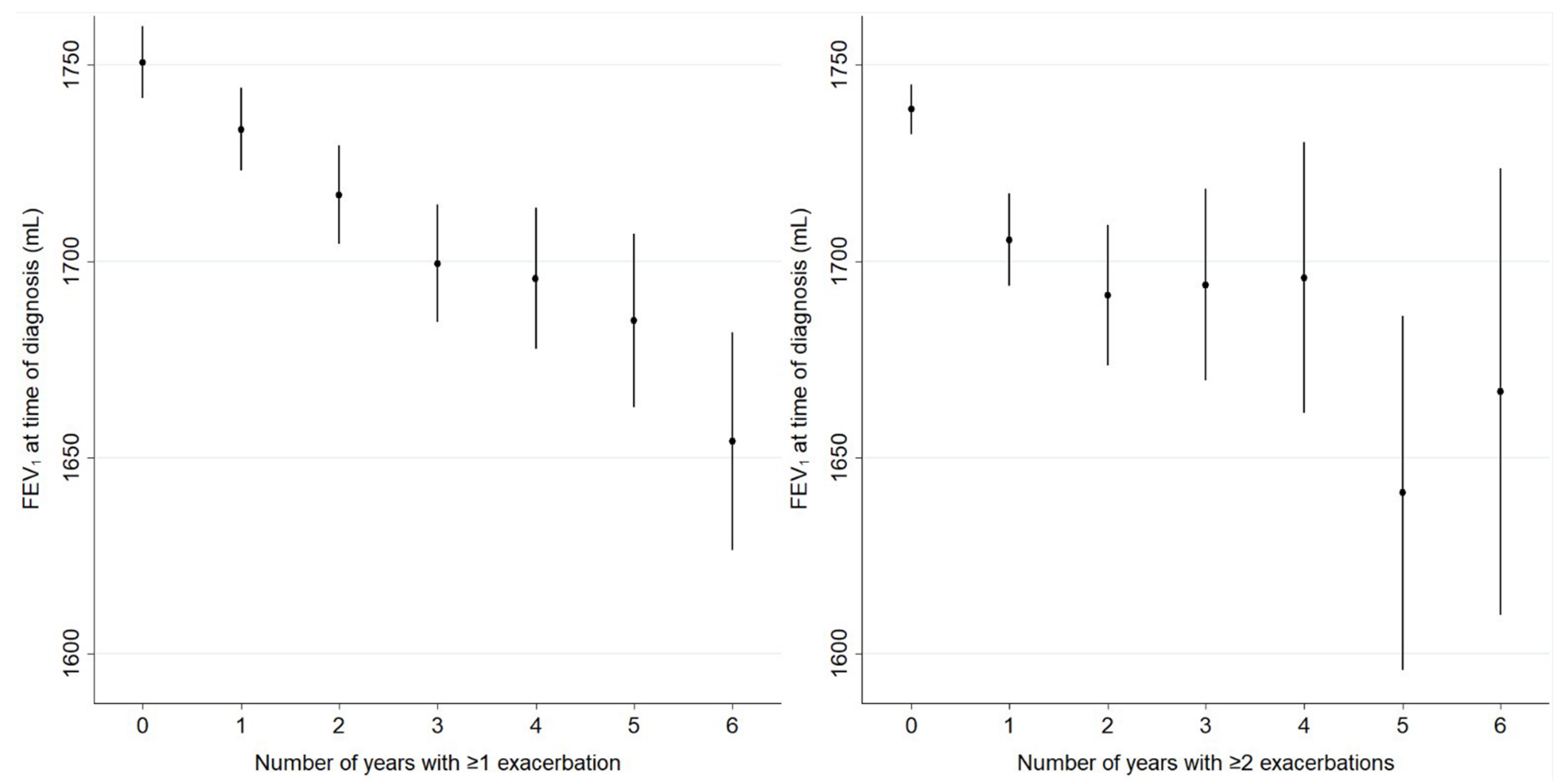

Figure 4 Marginal mean FEV $(\mathrm{mL})$ with $95 \%$ confidence interval at the time of first clinical COPD diagnosis by the number of years patients experienced $\geq 1$ (left) and $\geq 2$ (right) COPD exacerbations during 6 years after initiation of maintenance therapy.

mild to moderate COPD, ie, $\mathrm{FEV}_{1}$ was on average $15 \mathrm{~mL}$ lower with every additional year that patients experienced $\geq 1$ exacerbation after initiation of maintenance therapy. There was no evidence that treatment with ICS affects the association between lung function decline and exacerbation frequency.

Our findings suggest that the long-term exacerbation burden that patients experience despite receiving maintenance therapy is likely to be an important indicator for the clinical course of COPD. It may indicate the degree of underlying chronic airway inflammation, causing tissue destruction and progressive airflow restriction. Whether exacerbations cause excess decline or are the consequence of a period of more severe airway inflammation leading to potentially irreversible airflow restriction remains unclear. If inflammation does indeed play a key role in exacerbation, it will be pertinent to target inflammation at the earliest stages of the disease to change its clinical course.

Previous studies have reported an association between exacerbation frequency and lung function decline. ${ }^{11-14}$ A recent observational study demonstrated the association between acute exacerbations and lung function decline over 5 years to be greatest in patients with mild COPD (GOLD stage I), where each exacerbation was associated with an additional $23 \mathrm{~mL} /$ year decline $(95 \%$ confidence interval: 2 to 44$).{ }^{4}$ However, this was based on a relatively small population, and thus it was not possible to derive accurate estimates. The current study adds to our knowledge on the impact of increased COPD exacerbation burden, expressed as the number of years with exacerbation under maintenance therapy, on lung function decline in patients with mild-moderate COPD.

We observed a dose-response relationship between the number of years with exacerbations and lung function decline. Although the actual acceleration of $\mathrm{FEV}_{1}$ decline is relatively small for most patients with COPD who occasionally experience exacerbations, differences become relevant for long-term frequent exacerbators who do not seem to respond to maintenance therapy. For example, a patient aged 65 years at the time of COPD diagnosis, who had at least one exacerbation every year lost $9 \mathrm{~mL}$ extra above $31 \mathrm{~mL} /$ year, which is $29 \%$ more than similar patients without exacerbations. The associations may be stronger for severe exacerbations leading to hospitalization, which may be revealed by future studies.

\section{Strengths}

This study was conducted using two large primary care databases consisting of high-quality electronic medical records. This enabled research with a large sample size of COPD patients encompassing a long period of observation.

The utilization of real-life patient records also alludes to results that are more representative of the general COPD patient population, in contrast to Randomized Controlled 
trials (RCTs). Only a median of $23 \%$ patients with confirmed COPD diagnosis within the OPRCD would be eligible for inclusion to RCTs. ${ }^{15}$ This is in agreement with results from prior studies also reporting the low eligibility rate of the general COPD patient population for inclusion to RCTs. ${ }^{15-18}$

\section{Limitations}

There are several limitations of this study that warrant consideration. Firstly, we have no accurate information available whether $\mathrm{FEV}_{1}$ values were measured post-bronchodilation. Regardless, in the UK, the Quality and Outcomes Framework (QOF), a financial incentive system for the provision of quality primary care medical services within general practice, requires post-bronchodilator spirometry at the time of diagnosis and annual monitoring of spirometry. ${ }^{19}$ In the UK, spirometry should be quality assured and only be performed by people who have been trained and assessed to satisfy the standards set by the Association for Respiratory Technology and Physiology (ARTP) or equivalent. However, this framework to further improve the quality of diagnostic spirometry has only been introduced recently (September 2016) and, as of the writing of this manuscript, participation in spirometry training has not been made mandatory. Thus, quality of spirometry cannot be assured.

Secondly, like other observational studies using routinely collected patient data, this study is also susceptible to data inaccuracy and incompleteness ${ }^{20,21}$ within the OPCRD and CPRD, despite the extensive quality control and validity checks. The current study only reports associations without any judgment on the causal relationship between lung function decline and exacerbation. We also do not account for potential confounding due to the impact of changes in prescription within the follow-up period.

Lastly, the current study describes how doctors could use easily accessible information on the long-term burden of exacerbations to identify patients at risk of accelerated lung function decline, even before COPD has been diagnosed, and does not assess the impact of blood eosinophil counts and type of therapy on lung function decline. In a separate study, we report that COPD exacerbations are associated with a more rapid loss of lung function among patients with elevated blood eosinophils $(\geq 350$ cells $/ \mu \mathrm{L})$, when they are not treated with ICS therapy. ${ }^{22}$ This suggests a full blood count measurement to be the next step after spirometry for patients with a high exacerbation burden shows reduced lung function.

\section{Conclusion}

In conclusion, patients who experienced a greater exacerbation burden in spite of maintenance therapy had worse lung function when first diagnosed with mild to moderate COPD and had a more rapid lung function decline thereafter.

Our results stress the importance of optimal therapeutic interventions earlier in the course of the disease to reduce the underlying airway inflammation and to prevent irreversible airway remodeling leading to both exacerbations and fast lung function decline. Further research is required to understand whether lung function deterioration after exacerbation is permanent or partial reversibility of the lung function can be recouped with more aggressive and more effective treatment.

\section{Abbreviations}

COPD, chronic obstructive pulmonary disease; CPRD, Clinical Practice Research Datalink; FEV1, forced expiratory volume in 1 second; FVC, forced vital capacity; GOLD, global initiative for chronic obstructive lung disease; ICS, inhaled corticosteroid; IQR, interquartile range; LABA, long-acting $\beta 2$-agonists; LAMA, long-acting muscarinic antagonists; mMRC, modified medical research council; OPCRD, Optimum Patient Care Research Database; RCT, randomized control trial; $\mathrm{SD}$, standard deviation; UK, United Kingdom.

\section{Data Sharing Statement}

The dataset supporting the conclusions of this article was derived from the Clinical Practice Research Datalink (www. cprd.com) and the Optimum Patient Care Research Database (www.opcrd.co.uk). The CPRD has broad National Research Ethics Service Committee (NRES) ethics approval for purely observational research using the primary care data and established data linkages. The OPCRD has ethical approval from the National Health Service (NHS) Research Authority to hold and process anonymized research data (Research Ethics Committee reference: 15/EM/0150). This study was approved by the Anonymized Data Ethics Protocols and Transparency (ADEPT) committee - the independent scientific advisory committee for the OPCRD, and the Independent Scientific Advisory Committee (ISAC) for the CPRD. The authors do not have permission to give public access to the study dataset; researchers may request access to CPRD or OPCRD data for their own purposes. Access to CPRD can be made via the CPRD website (https://www.cprd.com/researcher/) or via the inquiries 
email enquiries@cprd.com. Access to OCPRD can be made via the OCPRD website (https://opcrd.co.uk/our-database /data-requests/) or via the inquiries email info@opcrd.co.uk.

\section{Ethics Approval}

OPCRD has received a favorable opinion for clinical research use from the Health Research Authority (REC reference: $15 / \mathrm{EM} / 0150)$. Governance is provided by the Anonymous Data Ethics Protocols and Transparency (ADEPT) committee, an independent body of experts and regulators commissioned by the Respiratory Effectiveness Group (REG, http://www.effectivenesseva luation.org/) to govern the standard of research conducted on internationally recognized databases. The study protocol was approved by the CPRD Independent Scientific Advisory Committee (ISAC approval number 17_117) and registered with the European Union electronic Register of Post-Authorization Studies (EU PAS Register number 19,879)

\section{Author Contributions}

All authors, including those employed by the funders of the study, participated in the data interpretation and writing of the report. All authors had full access to study results and had final responsibility for the decision to submit for publication. All authors made a significant contribution to the work reported, whether that is in the conception, study design, execution, acquisition of data, analysis and interpretation, or in all these areas; took part in drafting, revising or critically reviewing the article; gave final approval of the version to be published; have agreed on the journal to which the article has been submitted; and agree to be accountable for all aspects of the work.

\section{Funding}

This study is funded by AstraZeneca. AstraZeneca participated in the study design and reporting.

\section{Disclosure}

Marjan Kerkhof, Jaco Voorham, Victoria Carter and Janwillem W. H. Kocks are employees of the Observational and Pragmatic Research Institute, which conducted this study and which has conducted paid research in respiratory disease on behalf of the following other organizations in the past 5 years: Aerocrine, AKL Research and Development Ltd, Almirall, AstraZeneca, Boehringer Ingelheim, Chiesi,
GlaxoSmithKline, Mylan, Mundipharma, Napp, Novartis, Orion, Takeda, Teva, Zentiva (a Sanofi company). David B Price has board membership with Aerocrine, Amgen, AstraZeneca, Boehringer Ingelheim, Chiesi, Mylan, Mundipharma, Napp, Novartis, Regeneron Pharmaceuticals, Sanofi Genzyme, Teva Pharmaceuticals; consultancy agreements with Almirall, Amgen, AstraZeneca, Boehringer Ingelheim, Chiesi, GlaxoSmithKline, Mylan, Mundipharma, Napp, Novartis, Pfizer, Teva Pharmaceuticals, Theravance; grants and unrestricted funding for investigator-initiated studies (conducted through Observational and Pragmatic Research Institute Pte Ltd) from Aerocrine, AKL Research and Development Ltd, AstraZeneca, Boehringer Ingelheim, British Lung Foundation, Chiesi, Mylan, Mundipharma, Napp, Novartis, Pfizer, Regeneron Pharmaceuticals, Respiratory Effectiveness Group, Sanofi Genzyme, Teva Pharmaceuticals, Theravance, UK National Health Service, Zentiva (Sanofi Generics); payment for lectures/speaking engagements from Almirall, AstraZeneca, Boehringer Ingelheim, Chiesi, Cipla, GlaxoSmithKline, Kyorin, Mylan, Merck, Mundipharma, Novartis, Pfizer, Regeneron Pharmaceuticals, Sanofi Genzyme, Skyepharma, Teva Pharmaceuticals; payment for manuscript preparation from Mundipharma, Teva Pharmaceuticals; payment for the development of educational materials from Mundipharma, Novartis; payment for travel/accommodation/meeting expenses from Aerocrine, AstraZeneca, Boehringer Ingelheim, Mundipharma, Napp, Novartis, Teva Pharmaceuticals; funding for patient enrolment or completion of research from Chiesi, Novartis, Teva Pharmaceuticals, Zentiva (Sanofi Generics); stock/stock options from AKL Research and Development Ltd which produces phytopharmaceuticals; owns $74 \%$ of the social enterprise Optimum Patient Care Ltd (Australia and UK) and 74\% of Observational and Pragmatic Research Institute Pte Ltd (Singapore); and is peer reviewer for grant committees of the Efficacy and Mechanism Evaluation programme, and Health Technology Assessment. Claudia Cabrera, Paul Dorinsky and Patrick Darken are employees of AstraZeneca. Don D. Sin has received honoraria for speaking engagements from Boehringer Ingelheim (BI), AstraZeneca (AZ), Merck, and Novartis. He has received research funding from Merck, BI and AZ for work related to COPD and has sat on advisory boards of AZ, BI and Sanofi. Mohsen Sadatsafavi has received honoraria for speaking engagements from Boehringer Ingelheim, AstraZeneca, and GlaxoSmithKline. The authors report no other conflicts of interest in this work. 


\section{References}

1. Global strategy for the diagnosis, management, and prevention of COPD, Global Initiative for Chronic Obstructive Lung Disease (GOLD) 2020 Report. Available from: http://www.Goldcopd.Org/. Accessed February 2020.

2. Yeatts KB, Lippmann SJ, Waller AE, et al. Population-based burden of COPD-related visits in the ED: return ED visits, hospital admissions, and comorbidity risks. Chest. 2013;144(3):784-793. doi:10. 1378/chest.12-1899

3. Singh D, D'Urzo AD, Donohue JF, Kerwin EM. Weighing the evidence for pharmaco-logical treatment interventions in mild COPD; a narrative perspective. Respir Res. 2019;20(1):141. doi:10.1186/s12931-019-1108-9

4. Dransfield MT, Kunisaki KM, Strand MJ, et al. Acute exacerbations and lung function loss in smokers with and without chronic obstructive Pulmonary Disease. Am J Respir Crit Care Med. 2017;195 (3):324-330. doi:10.1164/rccm.201605-1014OC

5. Tantucci C, Modina D. Lung function decline in COPD. Int J Chron Obstruct Pulmon Dis. 2012;7:95-99. doi:10.2147/COPD.S27480

6. Bhatt SP, Soler X, Wang X, et al. Association between functional small airway disease and FEV1 decline in chronic obstructive pulmonary disease. Am J Respir Crit Care Med. 2016;194(2):178-184. doi:10.1164/rccm.201511-22190C

7. Optimum Patient Care Research Database (OPCRD). Available from: http://Optimumpatientcare.Org/Opcrd/. Accessed February 2020.

8. Quint JK, Mullerova H, DiSantostefano RL, et al. Validation of chronic obstructive pulmonary disease recording in the Clinical Practice Research Datalink (CPRD-GOLD). BMJ Open. 2014;4(7): e005540. doi:10.1136/bmjopen-2014-005540

9. Zafari Z, Sin DD, Postma DS, et al. Individualized prediction of lung-function decline in chronic obstructive pulmonary disease. CMAJ. 2016;188(14):1004-1011. doi:10.1503/cmaj.151483

10. Vestbo J, Anderson JA, Brook RD, et al. Fluticasone furoate and vilanterol and survival in chronic obstructive pulmonary disease with heightened cardiovascular risk (SUMMIT): a double-blind randomised controlled trial. Lancet. 2016;387(10030):1817-1826. doi:10.1016/S0140-6736(16)30069-1

11. Celli BR, Thomas NE, Anderson JA, et al. Effect of pharmacotherapy on rate of decline of lung function in chronic obstructive pulmonary disease: results from the TORCH study. Am J Respir Crit Care Med. 2008;178(4):332-338. doi:10.1164/rccm.200712-1869OC
12. Donaldson GC, Seemungal TA, Bhowmik A, Wedzicha JA. Relationship between exacerbation frequency and lung function decline in chronic obstructive pulmonary disease. Thorax. 2002;57 (10):847-852. doi: 10.1136/thorax.57.10.847

13. Makris D, Moschandreas J, Damianaki A, et al. Exacerbations and lung function de-cline in COPD: new insights in current and ex-smokers. Respir Med. 2007;101(6):1305-1312. doi:10.1016/j. rmed.2006.10.012

14. Halpin DMG, Decramer M, Celli B, Kesten S, Liu D, Tashkin DP. Exacerbation frequency and course of COPD. Int J Chron Obstruct Pulmon Dis. 2012;7:653-661. doi:10.2147/COPD.S34186

15. Halpin DM, Kerkhof M, Soriano JB, Mikkelsen H, Price DB. Eligibility of real-life patients with COPD for inclusion in trials of inhaled long-acting bronchodilator therapy. Respir Res. 2016;17 (1):120. doi:10.1186/s12931-016-0433-5

16. Kruis AL, Ställberg B, Jones RCM, et al. Primary care COPD patients compared with large pharmaceutically-sponsored COPD Studies: an UNLOCK validation study. PLoS One. 2014;9(3): e90145. doi:10.1371/journal.pone.0090145

17. Travers J, Marsh S, Caldwell B, et al. External validity of randomized controlled trials in COPD. Respir Med. 2007;101(6):1313-1320. doi:10.1016/j.rmed.2006.10.011

18. Herland K, Akselsen JP, Skjonsberg OH, Bjermer L. How representative are clinical study patients with asthma or COPD for a larger "real life" population of patients with obstructive lung disease? Respir Med. 2005;99(1):11-19. doi:10.1016/j.rmed.2004.03.026

19. Strong M, South G, Carlisle R. The UK quality and outcomes framework pay-for-performance scheme and spirometry: rewarding quality or just quantity? A cross-sectional study in Rotherham, UK. $B M C$ Health Serv Res. 2009;9:108. doi:10.1186/1472-6963-9-108

20. Wells BJ, Chagin KM, Nowacki AS, Kattan MW. Strategies for handling missing data in electronic health record derived data. EGEMS. 2013;1(3):1035.

21. van Staa T-P, Dyson L, McCann G, et al. The opportunities and challenges of pragmatic point-of-care randomised trials using routinely collected electronic records: evaluations of two exemplar trials. Health Technol Assess. 2014;18(43):1-146. doi:10.3310/hta18430

22. Kerkhof M, Voorham J, Dorinsky P, et al. Association between COPD exacerbations and lung function decline during maintenance therapy. Thorax. 2020. doi:10.1136/thoraxjnl-2019-214457

\section{Publish your work in this journal}

The International Journal of COPD is an international, peer-reviewed journal of therapeutics and pharmacology focusing on concise rapid reporting of clinical studies and reviews in COPD. Special focus is given to the pathophysiological processes underlying the disease, intervention programs, patient focused education, and self management protocols. This journal is indexed on PubMed Central, MedLine and CAS. The manuscript management system is completely online and includes a very quick and fair peer-review system, which is all easy to use. Visit http://www.dovepress.com/testimonials.php to read real quotes from published authors. 